\title{
Efficient Family of Iterative Methods for Solving Nonlinear Simultaneous Equations: A Comparative Study
}

\author{
Hazrat Ali ${ }^{1}$, Trishna Datta ${ }^{1}$, Md. Kamrujjaman ${ }^{2, *}$ \\ ${ }^{1}$ Department of Applied Mathematics, University of Dhaka, Dhaka 1000, Bangladesh. \\ ${ }^{2}$ Department of Mathematics, University of Dhaka, Dhaka 1000, Bangladesh.
}

How to cite this paper: Hazrat Ali, Trishna Datta, Md. Kamrujjaman. (2021) Efficient Family of Iterative Methods for Solving Nonlinear Simultaneous Equations: A Comparative Study. Journal of Applied Mathematics and Computation, 5(4), 331-337.

DOI: 10.26855/jamc.2021.12.011

Received: October 28, 2021

Accepted: November 23, 2021

Published: December 14, 2021

${ }^{*}$ Corresponding author: Md. Kamrujjaman, Department of Mathematics, University of Dhaka, Dhaka 1000, Bangladesh.

Email: kamrujjaman@du.ac.bd

\begin{abstract}
The solution of a system of nonlinear equations is presumably one of the most common but difficult features in numerical analysis in the sense of different aspirations for instance; high accuracy, minimum computation time, a small number of iterations along with less computational cost. In this study, four distinct iterative methods are presented for solving a system of nonlinear equations such as Broyden's method (BM), Optimal fourth-order method (OFOM), Optimal sixth order method (OSOM), and Homotopy continuation method (HCM). Detail formulations are explained along with the solution procedure. In addition, the rate of convergence and computational complexities are also explained within the formulations. Furthermore, to demonstrate and compare the efficiency of these methods, we solve two real-world practical nonlinear models. However, the results are presented numerically in a tabular form and the approximate results are compared with the exact and approximate solutions of other existing iterative methods. After analyzing the results, we conclude which method is better in what aspects.
\end{abstract}

\section{Keywords}

Broyden's method, Optimal fourth order method, Optimal sixth order method, Homotopy continuation method

\section{Introduction}

Usually, the solution of a system of nonlinear equations is required in the conclusive stage of real-world problems generated in mathematical physics, electrical engineering, robotics, mechanical and chemical engineering. Most of them are constructed based on various schemes such as Taylor series, variational iteration, quadrature formula, perturbation, and decomposition method; for instance, see the references [1-8]. Different methods have different amenities and limitations $[9,10]$. While some methods give good accuracy but take a large number of iterations [24]. On the other hand, some methods provide high accuracy in short iteration numbers but the computational complexity is immense which takes huge time to compute [25, 26, 27]. For this consequence, it is inevitable to compare these iterative methods from various aspects.

In this paper, we are going to present four iterative methods for solving a system of nonlinear equations such as Broyden's method, Optimal fourth and sixth-order methods, and Homotopy continuation method. After detailed formulation along with the application procedure, we will apply these methods to some practical models that arise in engineering. Finally, we will analyze the methods based on our experiments from different directions. 


\section{Mathematical formulation}

In this section, we will represent the derivations of four different iterative methods for the numerical solution of the system of nonlinear equations.

Let us consider a generalized form of the system of nonlinear equations as

$$
\begin{gathered}
f_{1}\left(x_{1}, x_{2}, \ldots, x_{n}\right)=0 \\
f_{2}\left(x_{1}, x_{2}, \ldots, x_{n}\right)=0 \\
\cdot \\
\cdot \\
f_{n}\left(x_{1}, x_{2}, \ldots, x_{n}\right)=0
\end{gathered}
$$

Assume that the system is solvable and its solution is $\left(x_{1}, x_{2}, \ldots, x_{n}\right)=\left(a_{1}, a_{2}, \ldots, a_{n}\right)$.

\subsection{Broyden's method}

Let us consider a system of nonlinear of the form $\mathrm{F}(\mathrm{X})=0$, where $F$ is a vector valued function of $\mathrm{X}$. Here $\mathrm{X}=\left(x_{1}, x_{2}, \ldots, x_{n}\right)$ and

$$
F(X)=\left(f_{1}\left(x_{1}, x_{2} \ldots, x_{n}\right), f_{2}\left(x_{1}, x_{2} \ldots, x_{n}\right), \ldots, f_{n}\left(x_{1}, x_{2} \ldots, x_{n}\right)\right) .
$$

Also let $X_{k}$ for $k=0$ is the initial condition and $\varepsilon_{1}, \varepsilon_{2}$ are two stopping criteria.

First of all, we should compute

$$
\Delta X_{k}=-\frac{F\left(X_{k}\right)}{J_{k}}
$$

where $J_{k}$ is the Jacobian computed as

$$
J_{k}=\left(\begin{array}{cccc}
\frac{\partial f_{1}\left(x_{1}, x_{2}, \ldots, x_{3}\right)}{\partial x_{1}} & \frac{\partial f_{1}\left(x_{1}, x_{2}, \ldots, x_{3}\right)}{\partial x_{2}} & \cdots & \frac{\partial f_{1}\left(x_{1}, x_{2}, \ldots, x_{3}\right)}{\partial x_{n}} \\
\frac{\partial f_{2}\left(x_{1}, x_{2}, \ldots, x_{3}\right)}{\partial x_{1}} & \frac{\partial f_{2}\left(x_{1}, x_{2}, \ldots, x_{3}\right)}{\partial x_{2}} & \cdots & \frac{\partial f_{2}\left(x_{1}, x_{2}, \ldots, x_{3}\right)}{\partial x_{n}} \\
\vdots & \vdots & \cdots & \vdots \\
\frac{\partial f_{n}\left(x_{1}, x_{2}, \ldots, x_{3}\right)}{\partial x_{1}} & \frac{\partial f_{n}\left(x_{1}, x_{2}, \ldots, x_{3}\right)}{\partial x_{2}} & \cdots & \frac{\partial f_{n}\left(x_{1}, x_{2}, \ldots, x_{3}\right)}{\partial x_{n}}
\end{array}\right)
$$

Then we should update by

$$
X_{k+1}=X_{k}+\Delta X_{k}
$$

Now the Jacobian will be updated by using previous results as

$$
J_{k+1}=J_{k}+\frac{\Delta F_{k}-J_{k} \Delta X_{k}}{\left\|X_{k}\right\|^{2}} X_{k}^{T}
$$

where $\Delta F_{k}=F\left(X_{k+1}\right)-F\left(X_{k}\right)$ Using the updated Jacobian in equation (2.1), we can find the most updated solution. Same procedure would be continued until

$$
\max || X_{k+1}-X_{k} \|<\varepsilon_{1}
$$

or

$$
\max || F\left(X_{k+1}\right)||<\varepsilon_{2}
$$

\subsection{Optimal 4th order method}

Let us consider a problem of nonlinear system $F(x)=0$ with nequations and nunknowns. There are several kinds 
of optimal fourth order method. Here we are considering the Optimal fourth order method combining Newton's and Traub's Method.

At first, we consider two step weighted-Newton method

$$
\begin{gathered}
y_{n}=x_{n}-\theta \frac{f\left(x_{n}\right)}{f^{\prime}\left(x_{n}\right)} \\
x_{n+1}=x_{n}-\left(\alpha+\beta \frac{f^{\prime}\left(x_{n}\right)}{f^{\prime}\left(y_{n}\right)}+\gamma \frac{f^{\prime}\left(y_{n}\right)}{f^{\prime}\left(x_{n}\right)}\right) \frac{f\left(x_{n}\right)}{f^{\prime}\left(x_{n}\right)}
\end{gathered}
$$

Here, $\alpha, \beta, \gamma, \theta$ are constants whose values determine the order of the method. To determine these values, let us consider the following theorem [11].

Theorem 1. [11] Let $f(x)$ be a real or complex function. Assuming that $\mathrm{f}(\mathrm{x})$ is sufficiently differentiable in an interval $\mathrm{I} \subseteq \mathrm{R}$, if $\mathrm{f}(\mathrm{x})$ has a simple root $\mathrm{x} \in \mathrm{I}$ and $\mathrm{x}_{0}$ is sufficiently close to root $\mathrm{X}$, then (2.2) has fourth order convergence if $\alpha=-\frac{1}{2}, \beta=\frac{9}{8}, \gamma=3 / 8$, and $\theta=\frac{2}{3}$.

Then the proposed Optimal fourth-order method becomes

$$
x_{n+1}=x_{n}-\left(\frac{-1}{2}+\frac{9}{8} \frac{f^{\prime}\left(x_{n}\right)}{f^{\prime}\left(y_{n}\right)}+\frac{3}{4} \frac{f^{\prime}\left(y_{n}\right)}{f^{\prime}\left(x_{n}\right)}\right) \frac{f\left(x_{n}\right)}{f^{\prime}\left(x_{n}\right)}
$$

Where $y_{n}=x_{n}-\frac{2}{3} \frac{f\left(x_{n}\right)}{f^{\prime}\left(x_{n}\right)}$

To apply this method for solving system of nonlinear equations, we have to follow the following procedure.

Let us consider the initial guess $x_{k}$ for $k=0$ and $\varepsilon$ be the tolerance. First of all, we should compute $y_{k}$ by using Newton's method as follow

$$
y_{k}=x_{k}-\frac{2}{3} F^{\prime}\left(x_{k}\right)^{-1} F\left(x_{k}\right) .
$$

Which will be updated again by Traud's method as

$$
z_{k}=x_{k}-F^{\prime}\left(x_{k}\right)^{-1}\left(F\left(x_{k}\right)+F\left(y_{k}\right)\right)
$$

The updated roots will then be calculated as

The procedure will be continued until ||$x_{k+1}-x_{k}||<\varepsilon$

$$
x_{k+1}=y_{k}-\left[F^{\prime}\left(z_{k}\right)\right]^{-1} F\left(y_{k}\right)
$$

\subsection{Optimal 6th order method}

Another optimal method for solving nonlinear system of equations $F(x)=0$ is Optimal Sixth-order method. And it is local order of convergence is six.

In this method, a new function calculation is required which is denoted by $J_{f}\left(x_{k}\right)$ and defined as,

Where $y_{k}=x_{k}-\frac{2}{3}\left(\frac{f\left(x_{k}\right)}{f^{\prime}\left(x_{k}\right)}\right)$

$$
J_{f}\left(x_{k}\right)=\frac{3 f^{\prime}\left(y_{k}\right)+f^{\prime}\left(x_{k}\right)}{6 f^{\prime}\left(y_{k}\right)-2 f^{\prime}\left(x_{k}\right)}
$$

Once again, we consider an initial guess $x_{k}$ for $k=0$ and a terminal condition $\varepsilon$. The beginning procedure, we should compute $y_{k}$ by using $x_{k}$ as $y_{k}=x_{k}-\frac{2}{3}\left(\frac{f\left(x_{k}\right)}{f^{\prime}\left(x_{k}\right)}\right)$

Further, the following iterations need to be performed

$$
J_{f}\left(x_{k}\right)=\frac{3 f^{\prime}\left(y_{k}\right)+f^{\prime}\left(x_{k}\right)}{6 f^{\prime}\left(y_{k}\right)-2 f^{\prime}\left(x_{k}\right)} z_{k}=x_{k}-J_{f}\left(x_{k}\right) \frac{f\left(x_{k}\right)}{f^{\prime}\left(x_{k}\right)}
$$

Finally, the updated roots will be calculated as

$$
x_{K+1}=z_{k}-\left(\left(f\left(z_{k}\right)\right) \times\left(\frac{3}{2} J_{f}\left(x_{k}\right) f^{\prime}\left(y_{k}\right)+\left(1-\frac{3}{2} J_{f}\left(x_{k}\right)\right) f^{\prime}\left(x_{k}\right)\right)^{-1}\right)
$$

This procedure will be repeated until ||$x_{k+1}-x_{k}||<\varepsilon$. 


\section{Homotopy Continuation Method}

In a similar manner, we consider a system of nonlinear equations such that $F(x)=0$ and $X$ be a solution of our problem. We are going to introduce a parameter $\lambda$ that take values from the interval $[0,1]$.

When $\lambda=0$, the solution $x_{0}$ is the initial solution. And the solution $x_{1}=X$ corresponding to $\lambda=1$ is the unknown solution of the system [12].

Suppose that the initial approximation $x_{0}$, the solution $F(X)=0$ is defined by

$$
\mathrm{T}:[0,1] \times \mathbb{R}^{n} \rightarrow \mathbb{R}^{n},
$$

where $\mathrm{T}(\lambda, X)=\lambda F(X)+(1-\lambda)\left[F(X)-F\left(X_{0}\right)\right]$.

We are going calculate a solution to

$$
\mathrm{T}(\lambda, X)=0
$$

for various values of $\lambda$. The function T provides us a family of functions with different values of $\lambda$ and this is going to lead us to the solution $X_{1}=X$. The function $\mathrm{T}$, between the function $\mathrm{T}(0, X)$ and $\mathrm{T}(1, X)$ is called a homotopy [12].

Now we are going to describe the continuation process. We assume an unique solution to the equation

$$
\mathrm{T}(\lambda, X)=0, \quad \lambda \in[0,1]
$$

If the functions $\lambda \rightarrow X(\lambda)$ and T are differentiable, then from equation (3.2), we have

$$
\begin{gathered}
\frac{\partial T(\lambda, X(\lambda))}{\partial \lambda}+\frac{\partial T(\lambda, X(\lambda))}{\partial X} X^{\prime}(\lambda)=0 \\
X^{\prime}(\lambda)=-\left[\frac{\partial T(\lambda, X(\lambda))}{\partial X}\right]^{-1} \frac{\partial T(\lambda, X(\lambda))}{\partial \lambda}
\end{gathered}
$$

Now we have a system of equations with an initial condition $X_{0}$. Since,

$$
\mathrm{T}(\lambda, X(\lambda))=F(X(\lambda))+(\lambda-1) F\left(X_{0}\right)
$$

So, we can determine both the Jacobian matrix $J(X(\lambda))$ and

$$
\frac{\partial T(\lambda, X(\lambda))}{\partial \lambda}=F\left(X_{0}\right)
$$

The system of differential equation becomes

$$
X^{\prime}(\lambda)=-[J(X(\lambda))]^{-1} F\left(X_{0}\right), \quad 0 \leq \lambda \leq 1 .
$$

This system of differential equations can be solved by Runge-Kutta method of fourth order [12].

\section{Numerical examples and applications}

System of nonlinear equations appear inside of many numerical computations [18-23]. In this section, we will consider the solution of some practical models that are generated in engineering and mathematical physics. The error norm is calculated as follows

$$
\|e\|_{2}=\sqrt{e_{1}^{2}+e_{2}^{2}+e_{3}^{2}+\ldots+e_{n}^{2}}
$$

\subsection{Chemical Model}

The following system of nonlinear equations represents a model of a chemical system that is used as a test system $[13,14]$. This system at steady state represents a model of two Continuous, Series, and non-adiabatic Tank Reactors (CSTR) with recycle and an exothermic irreversible reaction of order one. The equations are $[15,16]$

$$
\left\{\begin{array}{c}
(1-R)\left[\frac{D}{10\left(1+\beta_{1}\right)}-\Phi_{1}\right] \exp \left(\frac{10 \Phi_{1}}{1+\frac{10 \Phi_{1}}{\gamma}}\right)-\Phi_{1}=0 \\
(1-R)\left[\frac{D}{10}-\beta_{1} \Phi_{1}-\left(1+\beta_{2}\right) \Phi_{2}\right] \exp \left(\frac{10 \Phi_{2}}{1+\frac{10 \Phi_{2}}{\gamma}}\right)+\Phi_{1}-\left(1+\beta_{2}\right) \Phi_{2}=0
\end{array}\right.
$$




$$
0 \leq \Phi_{i} \leq 1, \quad i=1,2
$$

Here, we have two variables representing the reactor's temperature. They are respectively $\Phi_{1}$ and $\Phi_{2}$. In this system, $\beta_{1}, \beta_{2}, \gamma, D$ are constants and $R$ is the recycle ratio. For different values of $R$, the number of the solution vary. The initial condition for the problem is $(0.7,0.6)$. Exact result of the problem is $(0.724987,0.245240)$.

Table 1. Numerical comparison between Broyden's, Optimal 4th and 6th order and Homotopy continuation methods with $\left(R, D, \beta_{1}, \beta_{2}, \gamma\right)=(10,22,2,2,1000)$ of problem 4.1

\begin{tabular}{cccc}
\hline Methods & Iterations & $\operatorname{Roots}\left(\Phi_{1}, \Phi_{2}\right)$ & Error \\
\hline BM & 11 & $(0.72499,0.24524)$ & $1.604569 \times 10^{-07}$ \\
OFOM & 5 & $(0.724987,0.245241)$ & $8.956775 \times 10^{-07}$ \\
OSOM & 37 & $(0.724987,0.245240)$ & $2.311787 \times 10^{-03}$ \\
HCM & 200 & $(0.724987,0.245241)$ & $10^{-07}$ \\
\hline
\end{tabular}

This problem is solved by applying Broyden's method (BM), optimal fourth-order method (OFOM), optimal sixth-order method (OSOM), and Homotopy continuation method (HCM), and the numerical comparison is depicted in tabular form in Table 1. From the tabulated values, it is apparent that the optimal fourth-order method provides a good accuracy within a short iteration number, and the homotopy continuation method is computationally expensive.

\subsection{Thin wall problem}

This system of nonlinear equation is generated to find the solution of a thin wall rectangle girder section [16, 17].

$$
\left\{\begin{array}{l}
g_{1}(w, h, t)=w h+(w-2 t)(h-2 t)-165=0 \\
g_{2}(w, h, t)=\frac{w h^{3}}{12}-\frac{(w-2 t)(h-2 t)^{3}}{12}-9369=0 \\
g_{3}(w, h, t)=\frac{2(h-t)^{2}(w-t)^{2} t}{w+h-2 t}-6835=0
\end{array}\right.
$$

Here $w, h, t$ are the width, height and thickness of the rectangular section respectively, and $w, h, t \geq 0$.

Table 2. Numerical comparison between Broyden's, Optimal 4th and 6th order, and Homotopy continuation methods of problem 4.2

\begin{tabular}{cccc}
\hline Methods & Iterations & $\operatorname{Roots}(w, h, t)$ & Error \\
\hline BM & 17 & $(43.155566,10.128950,12.944048)$ & $3.639618 \times 10^{06}$ \\
OFOM & 5 & $(43.155566,10.128950,12.944048)$ & $1.595745 \times 10^{07}$ \\
OSOD & 50 & $(43.155566,10.128952,12.944050)$ & $2.365034 \times 10^{06}$ \\
HCM & 400 & $(43.155566,10.128950,12.944048)$ & $2.515823 \times 10^{03}$ \\
\hline
\end{tabular}

Similarly, we experiment with the methods that are discussed earlier to this real-world problem, and the results are presented in comparative tabular form (Table 2). The same consequences are noticed here as before which is that the optimal fourth iterative method is by far the most efficient compared to other methods that are discussed in this paper. It takes only 5 iterations to reach an accuracy of 6th decimal places.

\subsection{Complex problem} [12].

Finally, we consider a system of nonlinear problem that is comprised with trigonometric and exponential functions

$$
\left\{\begin{array}{c}
6 x_{1}-2 \cos \left(x_{2} x_{3}\right)-1=0 \\
9 x_{2}+\sqrt{x_{1}^{2}+\sin x_{3}+1.06}+0.9=0 \\
60 x_{3}+3 e^{-x_{1} x_{2}}+10 \pi-3=0
\end{array}\right.
$$

Using initial condition as $x^{0}=(0,0,0)^{t}$ and solving by prior discussed methods, we find the following results that is presented in Table 3. 
Table 3. Numerical comparison between Broyden's, Optimal 4th and 6th order, and Homotopy continuation methods of problem 4.3

\begin{tabular}{cccc}
\hline Methods & Iterations & Roots $\left(x_{1}, x_{2}, x_{3}\right)$ & Error \\
\hline BM & 5 & $(0.49814,-0.19961,-0.52883)$ & $8.1909 \times 10^{-09}$ \\
OFOM & 4 & $(0.49814,-0.19961,-0.52883)$ & $1.7224 \times 10^{-14}$ \\
OSOM & 15 & $(0.49815,-0.19961,-0.52883)$ & $5.6282 \times 10^{-07}$ \\
HCM & 2000 & $(0.49815,-0.19961,-0.52883)$ & $3.7921 \times 10^{-04}$ \\
\hline
\end{tabular}

\section{Concluding Remarks}

In this paper, we have presented detailed formulations of four iterative methods for solving a system of nonlinear equations. These methods are applied for solving two nonlinear models that are arisen in chemical and mechanical engineering. The numerical results are well presented in tabular form along with comparison. Here it is clear that the computational complexity of the Homotopy continuation method is immense. Also, both Broyden's, and optimal sixth-order methods provide better accuracy within a reasonable number of iterations. After analyzing the results, we can conclude that the optimal fourth-order method is the most efficient, computationally economical method.

\section{Acknowledgement}

The authors acknowledged to the anonymous reviewers for their suggestions to improve the quality of the manuscript. Author M. Kamrujjaman acknowledged to the University Grants Commission (UGC), Bangladesh.

\section{Conflict of Interest}

The authors declare no conflict of interest.

\section{References}

[1] Abbasbandy, S. (2003). Improving Newton-Raphson method for nonlinear equations by modified Adomian decomposition method. Applied Mathematics and Computation, 145(2-3), 887-893.

[2] Chun, C. and Ham, Y. (2007). A one-parameter fourth-order family of iterative methods for nonlinear equations. Applied Mathematics and Computation, 189(1), 610614.

[3] Daftardar-Gejji, V. and Jafari, H. (2006). An iterative method for solving nonlinear functional equations. Journal of Mathematical Analysis and Applications, 316(2), 753-763.

[4] He, J. H. (2003). A new iteration method for solving algebraic equations. Applied Mathematics and Computation, 135(1), 81-84.

[5] Aslam Noor, M. and Inayat Noor, K. (2006). Three-step iterative methods for nonlinear equations. Applied Mathematics and Computation, 183(1), 322-327.

[6] Noor, M. A., and Noor, K. I. (2006). Some iterative schemes for nonlinear equations. Applied Mathematics and Computation, 183(2), 774-779.

[7] Noor, M. A., Noor, K. I., and Waseem, M. (2010). Fourth-order iterative methods for solving nonlinear equations. International Journal of Applied Mathematics and Engineering Sciences, 4(1), 43-52.

[8] Ortega, J. M. and Rheinboldt, W. C. (2000). Iterative solution of nonlinear equations in several variables. Society for Industrial and Applied Mathematics.

[9] Broyden, C. G. (1965). A class of methods for solving nonlinear simultaneous equations. Mathematics of computation, 19(92), 577-593.

[10] Behl, R., Íñigo Sarría, Ruben Gonzalez Crespo, and Ángel Alberto Magreñán. (2019). Highly efficient family of iterative methods for solving nonlinear models. Journal of Computational and Applied Mathematics, 346, 110-132.

[11] Sharma, R. and Bahl, A. (2015). An optimal fourth order iterative method for solving nonlinear equations and its 
dynamics. Journal of Complex Analysis, 2015(8), 1-9.

[12] Burden, R. L. and Faires, J. D. (2005). Numerical analysis. 8th ed. Thomson Brooks/Cole.

[13] Hirsch, M. J., Pardalos, P. M., and Resende, M. G. (2009). Solving systems of nonlinear equations with continuous GRASP. Nonlinear Analysis: Real World Applications, 10(4), 2000-2006.

[14] Henderson, N., Sacco, W. F., and Platt, G. M. (2010). Finding more than one root of nonlinear equations via a polarization technique: An application to double retrograde vaporization. Chemical Engineering Research and Design, 88(5-6), 551-561.

[15] Floudas, C. A. (1999). Recent advances in global optimization for process synthesis, design and control: enclosure of all solutions. Computers and Chemical Engineering, 23, S963-S973.

[16] Pourjafari, E. and Mojallali, H. (2012). Solving nonlinear equations systems with a new approach based on invasive weed optimization algorithm and clustering. Swarm and Evolutionary Computation, 4, 33-43.

[17] Luo, Y. Z., Tang, G. J., and Zhou, L. N. (2008). Hybrid approach for solving systems of nonlinear equations using chaos optimization and quasi-Newton method. Applied Soft Computing, 8(2), 1068-1073.

[18] Ali, H., Kamrujjaman, M., and Shirin, A. (2021). Numerical solution of a fractional-order Bagley-Torvik equation by quadratic finite element method. Journal of Applied Mathematics and Computing, 66(1), 351-367.

[19] Akter, S. I., Mahmud, M. S., Kamrujjaman, M., and Ali, H. (2020). Global Spectral Collocation Method with Fourier Transform to Solve Differential Equations. GANIT: Journal of Bangladesh Mathematical Society, 40(1), 28-42.

[20] Ali, H. and Islam, M. S. (2017). Generalized Galerkin finite element formulation for the numerical solutions of second order nonlinear boundary value problems. GANIT: Journal of Bangladesh Mathematical Society, 37, 147-159.

[21] Ali, H., Kamrujjaman, M., and Islam, M. S. (2020). Numerical Computation of Fitzhugh-Nagumo Equation: A Novel Galerkin Finite Element Approach. International Journal of Mathematical Research, 9(1), 20-27.

[22] Ali, H., and Kamrujjaman, M. Numerical solutions of nonlinear parabolic equations with Robin condition: Galerkin approach.TWMS J. App. and Eng. Math. In press.

[23] Lima, S. A., Kamrujjaman, M., and Islam, M. S. (2021). Numerical solution of convection-diffusion-reaction equations by a finite element method with error correlation. AIP Advances, 11(8), 085225.

[24] Temelcan, G., Sivri, M., and Albayrak, I. (2020). A new iterative linearization approach for solving nonlinear equations systems. An International Journal of Optimization and Control: Theories \& Applications, 10(1), 47-54.

[25] Dehghan, M. and Shirilord, A. (2020). Three-step iterative methods for numerical solution of systems of nonlinear equations. Engineering with Computers, 1-14.

[26] Sayevand, K., Erfanifar, R., and Esmaeili, H. (2020). On Computational Efficiency and Dynamical Analysis for a Class of Novel Multi-step Iterative Schemes. International Journal of Applied and Computational Mathematics, 6(6), 1-23.

[27] Srivastava, H. M., Iqbal, J., Arif, M., Khan, A., Gasimov, Y. S., and Chinram, R. (2021). A new application of Gauss quadrature method for solving systems of nonlinear equations. Symmetry, 13(3), 432. 